\title{
Advances in cell-free biosensors: Principle, mechanism and applications
}

\author{
Liyuan Zhang ${ }^{1}$, Wei Guo ${ }^{2}$, and Yuan $\mathrm{Lu}^{1}$ \\ ${ }^{1}$ Tsinghua University \\ ${ }^{2}$ Shenyang Agricultural University
}

April 28, 2020

\begin{abstract}
Synthetic biology has promoted the development of biosensors as tools for detecting trace substances. In the past, biosensors based on synthetic biology have been designed on living cells, but the development of cell biosensors has been greatly limited by defects such as the obstruction of cell membrane. However, the advent of cell-free synthetic biology addresses these limitations. Biosensors based on the cell-free protein synthesis system have the advantages of higher safety, higher sensitivity, and faster response time over cell biosensors, which makes cell-free biosensors have a broader application prospect. This review summarizes the workflow of various cell-free biosensors, including the identification of analytes and signal output. The detection range of cell-free biosensors is greatly enlarged by different recognition mechanisms and output methods. In addition, the review also discusses the applications of cell-free biosensors in environmental monitoring and health diagnosis, as well as existing deficiencies and aspects that should be improved. In the future, through continuous improvement and optimization, the potential of cell-free biosensors will be stimulated, and their application fields will be expanded.
\end{abstract}

\section{Introduction}

Biosensors are analysis tools that take biological components as the main functional elements and used for rapid detection of various trace-level analytes. They generally consist of sensitive biorecognition elements, transducers, and signal analysis systems [1]. The sensitive element (enzyme, antibody, cell, etc. ) identifies and binds the analytes, and then the transducer captures the interaction between the sensitive material and the analyte and converts the analyte into an identifiable signal (e.g., electricity signal). The quantity of the analyte is further calculated through the signal analysis system. Compared with traditional instrument-based analytical chemistry such as gas chromatography (GC), high-performance liquid chromatography (HPLC), biosensors are less costly, more specific, faster, easier to operate, capable of on-line analysis, real-time identification, and simultaneous output of results. So they are more sensitive and more convenient. With the advantages of biosensors, they have been widely used in environmental detection, food safety inspection, disease diagnosis, biochemical analysis, and other fields [2-4].

Meanwhile, synthetic biology has propelled the development of biological sensors. Synthetic biology has great potential in the application of biosensors by designing genetic circuits and logic operations for biological components, resulting in a variety of genetic devices and biological modules [5]. It has promoted the construction of customized biological function system, which can finally realize sensors with customized programming sensitivity, specificity and dynamic range, and expand the scope of sensor detection target [6, 7], providing transformative tools for improving the performance of biosensors.

The design of synthetic biology is mainly dependent on living cells in recent decades, which leads to the rapid development of cell biosensors. Cell biosensors can use animal and plant cells, especially microbial cells as basal cells (sensitive element). Cells can metabolize a variety of compounds using intracellular enzymes with 
high stability and activity. Cell biosensors have a long service life and high reproducibility of experimental results [8]. Moreover, the sensitivity and specificity of biosensors can be improved by the introduction of corresponding genetic modules. Heavy metals, pesticides, vitamins, and some macromolecules can be detected using cell biosensors. For example, Hou et al. [9] specifically detected bioavailability of cadmium, lead, and arsenic in contaminated soil using a customized suite of multiple whole-cell Escherichia colisensors. Nevertheless, there are some shortcomings in cell biosensors that need to be addressed. Since cell biosensors use live genetically modified microorganisms (GMOs), if they are released into the environment, there are certain unsafe factors. In order to prevent the occurrence of this situation, it is usually impossible to apply these GMO biosensors [10, 11]. Furthermore, the response time of biosensors is prolonged because some analytes are hard to cross over the cell membrane. Moreover, some analytes may be toxic to living cells, so some cell-based biosensors are not suitable for detecting cytotoxic or impermeable membrane analytes, such as organophosphates. They may also be influenced by actual environmental samples as well as their own physiological and chemical conditions [12]. The above disadvantages lead to significant limitations for cell biosensors in applications.

However, the emergence of cell-free synthetic biology not only promotes the construction of cell-free biosensors in synthetic biology but also solves the limitations described above. Currently, the biosensor platform based on cell-free protein synthesis (CFPS) system has been developed [13]. The CFPS system is a mixed system of DNA templates, transcriptional machinery, translational machinery, the necessary substrates (including amino acids, energy substrates, cofactors, and salts), and water[14]. CFPS system can be divided into crude extract system and PURE system. The main difference between the two systems is the source of the translational machinery. As the name implies, one is from the crude extract, and the other one is from purified components. Compared with the crude extract system, the PURE system is more stable; however, the cost is higher, and the yield is also lower. The CFPS system and DNA template can be assembled into a mature cell-free biosensor. CFPS systems perform biological transcription and translation in an open environment, exhibiting a variety of unique functions superior to cellular systems and laying the foundation for biosensors to introduce more complex genetic circuits. Due to the uniqueness of the cell-free system, the biosensors based on the cell-free system have obvious advantages, which are reflected in the aspects of higher safety, higher tolerance, shorter response time, higher sensitivity, higher stability, and higher selectivity. These advantages are summarized in Table 1. Therefore, given these significant advantages, the application of cell-free systems in biosensor engineering is growing rapidly. The following contents focus on the design principle and workflow of cell-free biosensors, as well as the development of cell-free biosensors in environmental monitoring and biomedical applications.

\section{Principle and workflow of cell-free biosensors}

The design principle of most cell-free biosensors is to select the appropriate recognition mechanism and reporter gene based on the analytes, and then clone the encoded gene into the cell-free expression vector. Furthermore, select the appropriate cell-free protein synthesis system to constitute a mature cell-free biosensor with the coding template DNA. Cell-free biosensors can detect a variety of analytes, including ions, antibiotics, amino acids as well as nucleic acids of viral pathogens. The workflow of cell-free biosensor (Fig. 1) can be divided into two parts, the recognition and response of analytes by specific identification mechanisms, and the output of readable signals through reporter gene expression. First, accurate recognition of analytes is the key to cell-free biosensors. When analytes are added to the cell-free system, the sensor's recognition mechanism is regulated by specific molecular structure and binding $[15,16]$. Second, the downstream reporter gene is activated to be transcribed and translated into reporter proteins in a cell-free system. According to the presentation mode of different reporting proteins, the detection results are output by optical and other readable signals [17]. 


\subsection{Recognition-response mechanisms}

With the emergence of multiple analytes, some analytes are difficult to be recognized and detected by traditional recognition mechanisms (such as enzymes, antibodies, and PCR technology), which has led to the development of recognition mechanisms to use diversified molecular structures as recognition elements. The following contents mainly introduce the recognition mechanism of cell-free biosensors based on different molecular structures, including transcription factors, CRISPR-Cas, toehold switches, and adaptors [18].

\subsubsection{Transcription factor activation/repression}

A transcription factor (TF) is a protein molecule with a specific structure that binds to a specific gene and regulates gene expression at a specific intensity at a specific time and place. Therefore, the sensitivity and specificity of TFs can be used to design the sensor as a recognition element. TF-based cell-free biosensors have been used to identify specific small molecules, such as inorganic ions and organic molecules (bacterial quorum sensing molecules) [19], and they are also used in high-throughput screening and metabolic engineering [20]. Specific correct TFs or natural conformational TFs are selected by detecting different analytes, such as TF in response to aromatic compounds (XylS-AraC,XylR-NtrC, and LysR), metal ions (MerR, ArsR, DtxR, Fur, and NikR), or antibiotics (TetR and MarR) [21]. When analytes (ligands) are present in a cell-free environment, the response of TFs is immediately obtained, and binding of ligands to their activated TFs or the release of ligand-bound inhibitory TFs can lead to the reporter gene expression (Fig. 2A). For unknown ligands, they can be converted into detectable ligands by using metabolic enzymes [12].

To avoid the reaction of TFs to substances other than detection targets, TFs with enhanced specificity and sensitivity should be further expanded. Alternatively, a directed evolutionary strategy can be used to screen and select specific TFs [22]. Due to the known limitations of TFs, not all analytes can be identified, so there are other identification mechanisms [23, 24].

\subsubsection{Toehold switch and CRISPER-Cas recognition}

Cell-free biosensors based on toehold switch and CRISPER-Cas are mainly designed to detect genomic DNA or viral RNA of some pathogens. The toehold switch system is a programmable ribosome regulator, and it consists of two strands of RNA, called a switch or a trigger. The toehold switch sensor controls the translation of genes by triggering the binding of RNA through a trans effect. The switch contains a salient loop structure-forming in the upstream of the gene, which blocks gene translation in cis by blocking the ribosome binding site (RBS) [25]. After binding to the complementary trigger RNA, the sequestration is released, and the gene translation is activated [26]. The target nucleic acid is used as a trigger RNA to activate the expression of the reporter gene in cell-free system. Finally, the detection of the target can be determined directly by the expression of the reporter gene.

The cell-free biosensors based on CRISPER-Cas mainly rely on the cut principle of CRISPER-Cas to identify targets. CFPS system can express many kinds of active CRISPR machinery from plasmids or linear DNAs, and it can further output quantitative dynamics of gene cleavage or repression without the protein purification [27]. According to different Cas effectors (Cas9, Cas12a, and Cas13a), CRISPR biosensor systems have different shear cleavage principles [28]. For example, the CRISPER-Cas13a system can detect analytes that can directly cleave captured targets using the Cas13-crRNA complex [29], and then the lateral cracks of the complex are activated to cleave nearby non-targeted RNA (quenched fluorescent RNA). As a reporter, the quenched fluorescent RNA is cleaved and displays a fluorescence signal to respond to the results.

In addition, toehold switch and CRISPER-Cas can cooperate to detect analytes. For example, Pardee et al . [30] identified two strains by NASBA- CRISPER /Cas9 cleavage method (Fig. 2B). The nucleic acid sequence of a strain can be used as the target sequence. With the participation of reverse transcriptase and T7 RNA polymerase, the proto-spacer adjacent motifs (PAM) and trigger sequence (lacZ trigger) can be introduced in target sequence by isothermal amplification [31]. Cas endonuclease is mediated by sgRNA to 
recognize the PAM of the target sequence for fixed-point cutting. The uncut sequence contains the trigger RNA, which can be detected by the paper-based toehold switch sensor and finally identified as the strain type by the color change [32].

Nevertheless, there are some challenges to this method. The isothermal amplification process is susceptible to contamination, which could lead to off-target products and false positives. However, this phenomenon can be minimized by using sequentially specific fulcrum switch sensors, and CRISPR-Cas mediated amplification of downstream selection [33]. Multiplexing for CRISPR-Cas systems is also a considerable challenge [34, 35].

\subsubsection{Aptamer recognition}

Due to its small size, high stability, high affinity, and high specificity, aptamers can not only separate and combine with any selected molecule (including metal ions, small molecules, peptides, proteins, and even materials surfaces), but also can be easily modified in any position. Therefore, they are suitable for use as recognition elements of sensors $[36,37]$. Aptamers are single-stranded DNA or RNA molecules, most of which are obtained by a combinatorial biology technique called SELEX. In the cell-free environment, the singlestranded region of the aptamer is easily regulated by phage polymerase, which regulates the transcription and translation of the aptamer sequence through the T7 promoter. For example, Iyer et al . used nucleic acid aptamers to identify thrombin. They inserted thrombin-bound ssDNA aptamers in the downstream of the T7 promoter, creating a "bubble" structure downstream that binds to the aptamers in the presence of thrombin, blocking transcription of the T7 RNA polymerase (Fig. 2C). Finally, the presence of thrombin can be detected by the expression of the reporter gene [38, 39].

Although this method has high stability and specificity, its sensitivity needs to be further improved. In addition, the identification mechanism lacks high-quality aptamers for clinically important targets in medical applications. The aptamer technology must be extensively tested in clinical sample substrates to establish the reliability and accuracy.

\subsubsection{CFPS component identification}

In addition to the recognition mechanism mentioned above, there is another recognition mechanism that utilizes the cell-free translation system. So far, this mechanism has only been applied to amino acid detection. The design of a cell-free system selectively removes a specific amino acid, and it then adds the sample, which contains missing components from the system, such as amino acids in the patient's plasma sample [40] (Fig. 2D). Aminoacyl-tRNA synthase can be used as a recognition element to identify the amino acid in the sample and then produce aminoacyl tRNA. After the CFPS system is complete, the translation machinery is activated to produce protein output. Janget al . [41] used this principle to design cell-free biosensors to detect amino acids. This cell-free biosensor not only can accurately detect the amino acid content in the sample but also has a high sensitivity.

In addition to using the recognition mechanism of the cell-free translation system, most of the recognition mechanisms are encoded in the genetic circuit and then added to the cell-free hybrid system for characterization. Because the CFPS system can regulate the complex genetic circuits, it is possible to design a variety of recognition mechanisms for the detection of cell-free biosensors. This extends the types, levels, and ranges of target analytes to be detected.

\subsection{Signal output}

The signal of cell-free biosensors is mainly output in the form of optical or electrochemical signals. According to statistics, the optical reporter genes mainly include lux (bacterial luciferase),luc (firefly luciferase), gfp (green fluorescent protein), and lacZ ( $\beta$-galactosidase) [42]. After the identification, the downstream reporter gene is activated and expressed in the cell-free system. Various output methods are available for monitoring the proteins expressed by the reporter genes, including fluorescence, luminescence, visible color changes, etc.

The most common method is to report the fluorescence of proteins based on GFP which is a simple, convenient and intuitive signal output method in laboratory operation, and it does not require reaction substrates (Fig. 
3A). GFP expressed by the cell-free system could not glow by itself, and the fluorescence only can be generated by the light excitation. Therefore, the fluorescence output representing the gene expression level needs to be measured by means of absorbance detection. However, the obvious disadvantage of GFP is that the background signal is high, which makes the sensitivity of GFP low.

In addition, the signal can be output by the luciferase gene (lux/luc) (Fig. 3B). The luciferase experiment is extremely sensitive. It has no signal background and has a wide linear range (up to 7-8 orders of magnitude). Luciferase needs to be mixed with luciferin and other required chemicals to produce fluorescence. Generally, luciferase assay reagent is added to the test system, and the luciferase level is measured in the plate reader after the reactant mixing. Moreover, the application of luciferase also has certain limitations, such as short half-life, poor repeatability, and certain requirements on oxygen, etc.

Enzymes catalyze substrate molecules that can produce strong exponential signals than fluorescent proteins. $\beta$-galactosidase $(\beta$-gal) can lyse synthetic substrates, resulting in color changes, fluorescence, or chemiluminescence, which allows the output of LacZ-based sensors to be adjusted for different conditions. However, $\beta$-gal has some dependence on the substrate. The colorimetric method based on the enzyme activity of $\beta$-gal can directly observe the color changes with the naked eye, and the most common substrate for colorimetric detection is O-nitrophenyl-d-pyrine galactoside. This signal output method is suitable for paper-based cellfree biosensors (Fig. 3C). Because the cell-free biosensors based on luciferases and $\beta$-gal have high sensitivity and short response time, so luc and lacZ are preferred reporter genes.

Except for the optical signal output, cell-free biosensors can also be output by electrochemical measurement. A variety of material electrodes combined with cell-free system and electrochemical signals can be generated, while the electrodes can capture the target. Capacitance-voltage and constant-capacitance measurements are employed to monitor the capacitance changes induced by surface modification steps for sensor preparation as well as interactions between receptor and its ligands [43]. Mousavi et al . [44] designed an extensible reporter enzyme system, in which a sensor based on a cell-free gene circuit cleaves specific DNA sequences in solution, generating electrochemical signals when the newly released strands are trapped on the surface of nanostructured microelectrodes (Fig. 3D). This method is in sharp contrast to the traditional optical reporter, which not only has limited multiplexing ability but also has a dependence on ventilation and reduced sensitivity in turbid solutions [45]. These limitations can be overcome by electrochemical measurements. The electrochemical system will enable the multiplexing of gene-based circuits for portable detection and will facilitate the development of electronic devices related to synthetic biology.

\section{Improvement of cell-free biosensors in practical application}

To keep up with the development of practical applications, the cell-free biosensor is required to be more portable, stable, and cost-efficient. These requirements have led to continuous improvement of cell-free biosensors. Generally, cell-free biosensor reagents are often refrigerated or frozen during storage or transportation. This method of storage is not convenient, which may hinder the field deployment. Therefore, the researchers develop the lyophilization technology to improve the storage stability of cell-free biosensors. The cell-free system ensures that it remains active after lyophilization and does not adversely affect the efficiency. By freeze-drying, the cold-chain storage problems can be overcome to stabilize the long-term and higher temperature storage of the cell extract, which can last up to three months to a year and reduce the necessary storage space. Moreover, the stability of cell-free biosensors can be improved in other ways. For example, Karig et al. [46] also investigated using the non-reducing disaccharide trehalose to protect cell-free components during oven drying. Moreover, components can be encapsulated in the liposomes [47] or polymer substrates to maintain the system stability [48]. However, the decrease of the activity of the cell-free system after lyophilization is the main improvement target that needs to be developed in the future.

To facilitate the field monitoring, biosensors need improving the portability. Cell-free systems can be freezedried with appropriate supports (e.g., microtubules and microporous plates). Beyond that, the cell-free system also can be introduced onto a cheap matrix simple paper [26], where the freeze-drying and the signal amplification can reduce the chance of contamination and false positives. Because of the small size 
of the freeze-dried reagent required for the paper-based cell-free biosensor, this greatly reduces the cost and facilitates the field testing.

Furthermore, there may be some differences in the cell-free systems prepared in different batches, so it is necessary to standardize the cell-free systems. PURE systems can be developed to improve sensor standards. The PURE system for cell-free protein synthesis reduces the level of contaminated proteases, nucleases, and phosphatases, and provides a more precise method of preparation, a higher reproducibility, and better flexibility of the modular system. Because there is no metabolic side effect of amino acid library like in crude extract-based cell-free system, so the PURE system is more stable [49]. The PURE system has been commercialized to make them available for a variety of applications, with strict quality control. However, the commercial PURE system is expensive, and the production cost of the PURE system is very high, so the general sensor design still uses the crude extract system. Therefore, in order to standardize the cell-free biosensors, more time and energy should be spent on the development of the PURE system to improve the efficiency and reduce the cost in the future. At present, there is some lag in commercial applications of cell-free biosensors, which will be the focus of future development.

\section{Applications}

At present, we are all faced with the situation of frequent environmental pollution and human diseases. All kinds of environmental pollutants and diseases seriously threaten the living environment and health of human beings. To detect these hazards more quickly and accurately, biosensors with low cost and high efficiency are urgently needed. In this context, cell-free biosensors have made remarkable achievements in the rapid detection of environmental pollutants and disease pathogens (Fig. 4).

\subsection{Environmental detection}

Many chemical pollutants exist in the environment, which pose some serious threats because they are invisible or hard to spot. The most common harmful substances are heavy metals, such as arsenic and mercury[50], as well as toxic small molecules and antibiotics [51, 52]. The environmental protection agency (EPA) stipulates that some harmful pollutants cannot exceed a certain level in the environment, so it is necessary to test the present level of these substances in the environment. A number of cell-free biosensors have been developed and designed to detect the presence or content of hazardous substances in environmental samples.

When inorganic heavy metals enter the body from the environment, they accumulate in certain organs of the body, cause chronic poisoning, and harm health. Researchers have designed a number of cell-free biosensors for detecting heavy metals. For example, a paper-based mercury biosensor was designed by the iGEM team Peking 2010 [53]. By adjusting the plasmid concentrations and adding new translation enhancement sequences to optimize the cell-free mercury sensor, it increased the yield of the CFPS reaction and enabled accurate quantification of the analytes. It can detect $6 \mu \mathrm{g} / \mathrm{L}$ of mercury concentration. The paper-based cell-free biosensor has a low cost and is convenient for detection. In addition, the team built a 3D-printed box that can be connected to a phone for real-time readings, extending the detection platform to real-time applications.

There are also some cell-free biosensors designed to detect toxic organic molecules. For instance, Khalidet al . [53] developed a cell-free biosensor platform, a ligand-induced activated RNA output sensor (ROSALIND), which uses a variety of TFs to detect harmful pollutants in water samples, such as toxic PPCP compounds (salicylate, benzalkonium chloride and uric acid). However, when testing non-laboratory samples (e.g., drinking water and lake water), the sensitivity of the sensor may be reduced due to the interference of the molecular components in the samples. Therefore, the matrix effect of these test samples needs to be further explored in future work.

The presence of antibiotics in the environment is harmful to the ecological balance and human health. Therefore, its presence in the environment needs to be monitored in a timely manner. Duyen et al . [54] have developed a simple biological sensor based on color paper, inhibition of bacteria used in the detection of protein synthesis of antibiotics. The detection limits of paramycin, tetracycline, erythromycin, 
and chloramphenicol were further estimated to be $0.5,2.1,0.8$, and $6.1 \mu \mathrm{g} / \mathrm{mL}$. Pellinen et al . [55] designed a novel cell-free biosensor for the detection of tetracycline hydrochloride $(\mathrm{Tc}-\mathrm{HCl})$. $\mathrm{Tc}-\mathrm{HCl}$ has been detected at concentrations below $10 \mathrm{ng} / \mathrm{ml}$. The maximum residue limit for Tc-HCl in EU milk samples is $100 \mathrm{ng} / \mathrm{ml}$, and the limit for $\mathrm{Tc}-\mathrm{HCl}$ in commercial microbial inhibition tests is 30 to $500 \mathrm{ng} / \mathrm{ml}$. It can be seen that the detection limit of cell-free biosensors can reach the ppt level, which has reached the environmental standard level for the detection of some pollutants. The test results can be used to judge whether the samples under test meet the standards or not. Although the sensitivity of current cell-free biosensors can reach a certain level, some substances with lower detection limits still exist or will appear in the future, which requires us to continuously improve and optimize the cell-free biosensors.

These examples demonstrate that cell-free biosensors have been successfully applied to the detection of toxic substances in the environment. Due to its low detection limit and fast and convenient characteristics, cell-free biosensors can quickly detect pollutants, determine the safety limit of analysis samples, and timely monitor the environment for timely prevention or repair. Through the continuous optimization of cell-free biosensors, it has a broad prospect in environmental monitoring.

\subsection{Clinical biomedical applications}

Because of the high sensitivity and fast response time of cell-free biosensors, they can quickly judge the diagnosis result, which occupies an important position in biomedicine. The existing cell-free biosensors mainly focus on the detection of clinically relevant carcinogens, bacteria, and pathogens (e.g., EDCs, AA, and AHL) [17, 56-58]. These sensors can be used to screen pathogens that can be detected in blood, urine, and sputum samples from clinical patients.

The well-known chemicals called endocrine-disrupting chemicals (EDCs) [59,60], widely exist in the environment, food, and personal care products. Exposure to EDCs can lead to acute and chronic diseases, including cancer and diabetes. Salehi et al . [61] designed a hER $\beta$-CFPS biosensor to detect endocrine xenoestrogens (XEs, one kind of the EDCs) in human blood and urine. Because these test samples (blood, urine, and sputum samples from clinical patients) are complex, they can interfere with the cell-free system to a certain degree and may alter the delicate calibration of protein synthesis reactions, making the test process less smooth. In order to solve this problem, some measurements can be taken to pretreat the sample. They added RNA enzyme inhibitors to the samples, which can reduce the impact of urine or blood samples on the CFPS system. This can simplify the detection process, which can be used for more complex analytes and expand the application range of cell-free biosensors.

The virus is rapidly pathogenic, and some are highly infectious. The severity of the virus infection and its unknown complications make timely diagnosis of the virus critical to the human health, which also can limit the rapid spread of the virus. In this context, some cell-free biosensors are designed to detect viruses. Gootenberg et al . [62] developed a rapid diagnostic cell-free biosensor (SHERLOCK) based on CRISPR-Cas13a to detect Zika virus in human serum samples. The sensitivity of the sensor is similar to that of ddPCR and qPCR, but with less variation. To improve the specificity of the sensor, they synthesized mismatched crRNA, which allowed them to distinguish between different strains (Zika virus and dengue fever). Cell-free biosensors can also be used to detect other viruses, such as Ebola virus. Now there is a kind of electrochemical DNA biosensor for detecting examples of this kind of virus [63]. In a word, cell-free biosensors can detect not only the presence or absence of a single virus but also different types of viruses.

The content of amino acids also has a great impact on human health. The lack of amino acids can lead to abnormal physiological functions, affect the normal progress of antibody metabolism, and lead to diseases. It can be used as an early detection method for several types of cancer. Janget al . [41, 64] designed a CFPS biosensor to detect amino acids in fetal bovine serum (FBS) samples. The amino acid content was successfully detected, and the detection limit was less than $100 \mathrm{nM}$. However, the types of amino acids detected by cell-free sensors based on crude extracts are limited, so they suggested using the PURE system for cell-free biosensors. It can detect more kinds of amino acids (Asp, Asn, Glu, and Gln) than the crude extract system, and the detection threshold is lower [65]. 
These examples show that cell-free biosensors have significant advantages in medical diagnosis and clinical sample testing. They can be used for early detection of disease and also can respond quickly to virus outbreaks or the occurrence of genetic variations and mutations to help monitor the spread of disease.

\section{Conclusion}

The cell-free system with unique advantages provides a novel design platform for the development of biosensors. Considering the openness and rapid prototyping capability of CFPS, the CFPS system can open up more design space for the construction and optimization of biosensors and genetic circuits. Cell-free biosensors based on TF, RNA, and toehold switch can reduce the number of cycles required to design and help build complex genetic networks. CFPS system can also be integrated with mathematical modeling and high technologies (e.g., automation and microfluidics) to unlock the potential of cell-free biosensors.

In the practical applications of cell-free biosensors, the lyophilization technology based on the portable paper platform can maintain the stability of the system, which promotes its portable use and reduces the cost. However, there are still many challenges to be addressed. The decline of the activity after the storage is a problem that needs to be further improved. To improve in situ detection, multiplexing functions, the sensitivity, and the specificity of cell-free biosensors need to be further optimized.

In addition, cell-free biosensors can use CFPS systems other than E. coli, such as yeast and mammalian cells, to develop more sophisticated biosensors. By improving the sensor recognition mechanisms and combining cell-free systems with other materials (e.g., silicon), more types of functional cell-free biosensors can be developed. It might expand the detection range of cell-free biosensors to sense the odor, air, temperature, light, and osmotic pressure. With further evolution of cell-free biosensors, besides environmental monitoring and human health diagnosis, they will increasingly expand their applications to food testing, classroom education, and others, making them more practical and commercial.

\section{Acknowledgment}

This work was supported by the National Key R\&D Program of China (Grant No. 2018YFA0901700), the Institute for Guo Qiang, Tsinghua University (Grant No. 2019GQG1016), and the Laboratory Innovation Fund of Tsinghua University.

\section{Conflict of interest}

The authors declare no financial or commercial conflict of interest.

\section{Reference}

[1] Perumal, V. and U. Hashim, Advances in biosensors: Principle, architecture and applications. Journal of Applied Biomedicine, 2014. 12(1): p. 1-15.

[2] Misawa, N., T. Osaki, and S. Takeuchi, Membrane protein-based biosensors. Journal of The Royal Society Interface, 2018. 15(141): p. 20170952.

[3] Borisov, S.M. and O.S. Wolfbeis, Optical biosensors.Chemical reviews, 2008. 108(2): p. 423-461.

[4] Castillo, J., S. Gáspár, S. Leth, M. Niculescu, et al.,Biosensors for life quality: Design, development and applications. Sensors and Actuators B: Chemical, 2004. 102(2): p. 179-194.

[5] Khalil, A.S. and J.J. Collins, Synthetic biology: applications come of age. Nature Reviews Genetics, 2010. 11(5): p. 367-379.

[6] Kim, H.J., H. Jeong, and S.J. Lee, Synthetic biology for microbial heavy metal biosensors. Analytical and bioanalytical chemistry, 2018. 410(4): p. 1191-1203.

[7] Konig, H., D. Frank, R. Heil, and C. Coenen, Synthetic genomics and synthetic biology applications between hopes and concerns.Current genomics, 2013. 14(1): p. 11-24. 
[8] Audrey, S., P.-S. Beatriz, and M. Jean-Louis, Biosensors for pesticide detection: new trends. American Journal of Analytical Chemistry, 2012. 2012.

[9] Hou, Q., A. Ma, T. Wang, J. Lin, et al., Detection of bioavailable cadmium, lead, and arsenic in polluted soil by tailored multiple Escherichia coli whole-cell sensor set. Analytical and bioanalytical chemistry, 2015. 407(22): p. 6865-6871.

[10] Kviatkovski, I., S. Shushan, Y. Oron, I. Frumin, et al.,Smelling Pseudomonas aeruginosa infections using a whole-cell biosensor-An alternative for the gold-standard culturing assay.Journal of biotechnology, 2018. 267: p. 45-49.

[11] Schirmer, C., J. Posseckardt, A. Kick, K. Rebatschek, et al.,Encapsulating genetically modified Saccharomyces cerevisiae cells in a flow-through device towards the detection of diclofenac in wastewater. Journal of biotechnology, 2018. 284: p. 75-83.

[12] Voyvodic, P.L. and J. Bonnet, Cell-free biosensors for biomedical applications. Current Opinion in Biomedical Engineering, 2019.

[13] Pandi, A., I. Grigoras, O. Borkowski, and J.-L. Faulon, Optimizing Cell-Free Biosensors to Monitor Enzymatic Production.ACS Synthetic Biology, 2019. 8(8): p. 1952-1957.

[14] Carlson, E.D., R. Gan, C.E. Hodgman, and M.C. Jewett,Cell-free protein synthesis: applications come of age.Biotechnology advances, 2012. 30(5): p. 1185-1194.

[15] Taylor, N.D., A.S. Garruss, R. Moretti, S. Chan, et al.,Engineering an allosteric transcription factor to respond to new ligands. Nature methods, 2016. 13(2): p. 177.

[16] Pfeiffer, F. and G. Mayer, Selection and biosensor application of aptamers for small molecules. Frontiers in chemistry, 2016. 4: p. 25.

[17] Voyvodic, P.L., A. Pandi, M. Koch, I. Conejero, et al.,Plug-and-play metabolic transducers expand the chemical detection space of cell-free biosensors. Nature communications, 2019. 10(1): p. 1-8.

[18] Kim, D.-M. and K.-H. Lee, In vitro use of cellular synthetic machinery for biosensing applications. Frontiers in pharmacology, 2019. 10: p. 1166.

[19] Snoek, T., E. Chaberski, F. Ambri, S. Kol, et al.,Evolution-guided engineering of small-molecule biosensors. Biorxiv. 2019 .

[20] Zhang, J., M.K. Jensen, and J.D. Keasling, Development of biosensors and their application in metabolic engineering. Current opinion in chemical biology, 2015. 28: p. 1-8.

[21] Cheng, F., X.L. Tang, and T. Kardashliev, Transcription factor-based biosensors in high-throughput screening: advances and applications. Biotechnology journal, 2018. 13(7): p. 1700648.

[22] D'ambrosio, V. and M.K. Jensen, Lighting up yeast cell factories by transcription factor-based biosensors. FEMS yeast research, 2017. 17(7).

[23] Fernandez-Lopez, R., R. Ruiz, F. de la Cruz, and G. Moncalian,Transcription factor-based biosensors enlightened by the analyte.Frontiers in microbiology, 2015. 6: p. 648.

[24] Mahr, R. and J. Frunzke, Transcription factor-based biosensors in biotechnology: current state and future prospects.Applied microbiology and biotechnology, 2016. 100(1): p. 79-90.

[25] Green, A.A., P.A. Silver, J.J. Collins, and P. Yin, Toehold switches: de-novo-designed regulators of gene expression.Cell, 2014. 159(4): p. 925-939.

[26] Pardee, K., A.A. Green, T. Ferrante, D.E. Cameron, et al.,based synthetic gene networks. Cell, 2014. 159(4): p. 940-954. 
[27] Marshall, R., C.S. Maxwell, S.P. Collins, T. Jacobsen, et al.,Rapid and scalable characterization of CRISPR technologies using an E. coli cell-free transcription-translation system. Molecular cell, 2018. 69(1): p. 146-157. e3.

[28] Li, Y., S. Li, J. Wang, and G. Liu, CRISPR/Cas systems towards next-generation biosensing. Trends in biotechnology, 2019. 37(7): p. 730-743.

[29] Myhrvold, C., C.A. Freije, J.S. Gootenberg, O.O. Abudayyeh, et al., Field-deployable viral diagnostics using CRISPR-Cas13.Science, 2018. 360(6387): p. 444-448.

[30] Pardee, K., A.A. Green, M.K. Takahashi, D. Braff, et al.,Rapid, low-cost detection of Zika virus using programmable biomolecular components. Cell, 2016. 165(5): p. 1255-1266.

[31] Casper, E.T., S.S. Patterson, P. Bhanushali, A. Farmer, et al., A handheld NASBA analyzer for the field detection and quantification of Karenia brevis. Harmful Algae, 2007. 6(1): p. 112-118.

[32] Chen, J.S., E. Ma, L.B. Harrington, M. Da Costa, et al.,CRISPR-Cas12a target binding unleashes indiscriminate single-stranded DNase activity. Science, 2018. 360(6387): p. 436-439.

[33] Cordray, M.S. and R.R. Richards-Kortum, Emerging nucleic acid-based tests for point-of-care detection of malaria. The American journal of tropical medicine and hygiene, 2012. 87(2): p. 223-230.

[34] Bruch, R., G.A. Urban, and C. Dincer, CRISPR/Cas Powered Multiplexed Biosensing. Trends in biotechnology, 2019. 37(8): p. 791-792.

[35] Li, Y., L. Liu, and G. Liu, CRISPR/Cas multiplexed biosensing: a challenge or an insurmountable obstacle? Trends in biotechnology, 2019. 37(8): p. 792-795.

[36] Song, S., L. Wang, J. Li, C. Fan, et al., Aptamer-based biosensors. TrAC Trends in Analytical Chemistry, 2008. 27(2): p. 108-117.

[37] Zhou, W., P.-J.J. Huang, J. Ding, and J. Liu,Aptamer-based biosensors for biomedical diagnostics. Analyst, 2014. 139(11): p. 2627-2640.

[38] Iyer, S. and M.J. Doktycz, Thrombin-mediated transcriptional regulation using DNA aptamers in DNAbased cell-free protein synthesis. ACS synthetic biology, 2014. 3(6): p. 340-346.

[39] Wang, J., L. Yang, X. Cui, Z. Zhang, et al., A DNA bubble-mediated gene regulation system based on thrombin-bound DNA aptamers. ACS synthetic biology, 2017. 6(5): p. 758-765.

[40] Catherine, C., S.J. Oh, K.H. Lee, S.E. Min, et al.,Engineering Thermal Properties of Elastin-like Polypeptides by Incorporation of Unnatural Amino Acids in a Cell-free Protein Synthesis System. Biotechnology \& Bioprocess Engineering. 20(3): p. 417-422.

[41] Jang, Y.-J., K.-H. Lee, T.H. Yoo, and D.-M. Kim, Complementary cell-free translational assay for quantification of amino acids. Analytical chemistry, 2017. 89(18): p. 9638-9642.

[42] Gui, Q., T. Lawson, S. Shan, L. Yan, et al., The application of whole cell-based biosensors for use in environmental analysis and in medical diagnostics. Sensors, 2017. 17(7): p. 1623.

[43] Bronder, T.S., A. Poghossian, S. Scheja, C. Wu, et al.,DNA immobilization and hybridization detection by the intrinsic molecular charge using capacitive field-effect sensors modified with a charged weak polyelectrolyte layer. ACS applied materials \& interfaces, 2015. 7(36): p. 20068-20075.

[44] Mousavi, P.S., S.J. Smith, J.B. Chen, M. Karlikow, et al., A multiplexed, electrochemical interface for gene-circuit-based sensors. Nature chemistry, 2020. 12(1): p. 48-55.

[45] Biran, I., R. Babai, K. Levcov, J. Rishpon, et al.,Online and in situ monitoring of environmental pollutants: electrochemical biosensing of cadmium. Environmental Microbiology, 2000. 2(3): p. 285-290. 
[46] Karig, D.K., S. Bessling, P. Thielen, S. Zhang, et al.,Preservation of protein expression systems at elevated temperatures for portable therapeutic production. Journal of The Royal Society Interface, 2017. 14(129): p. 20161039.

[47] Schwarz-Schilling, M., L. Aufinger, A. Muckl, and F. Simmel, Chemical communication between bacteria and cell-free gene expression systems within linear chains of emulsion droplets.Integrative Biology, 2016. 8(4): p. 564-570.

[48] Lim, S.Y., K.-O. Kim, D.-M. Kim, and C.B. Park,Silica-coated alginate beads for in vitro protein synthesis via transcription/translation machinery encapsulation. Journal of biotechnology, 2009. 143(3): p. 183-189.

[49] Whittaker, J.W., Cell-free protein synthesis: the state of the art. Biotechnology letters, 2013. 35(2): p. 143-152.

[50] Grawe, A., A. Dreyer, T. Vornholt, U. Barteczko, et al.,A paper-based, cell-free biosensor system for the detection of heavy metals and date rape drugs. PloS one, 2019. 14(3).

[51] Zhang, P., H. Feng, J. Yang, H. Jiang, et al., Detection of inorganic ions and organic molecules with cell-free biosensing systems. Journal of biotechnology, 2019. 300: p. 78-86.

[52] Liu, X., A.D. Silverman, K.K. Alam, E. Iverson, et al.,Design of a transcriptional biosensor for the portable, on-demand detection of cyanuric acid. ACS Synthetic Biology, 2019.

[53] Alam, K.K., J.K. Jung, M.S. Verosloff, P.R. Clauer, et al., Rapid, low-cost detection of water contaminants using regulated in vitro transcription. BioRxiv, 2019: p. 619296.

[54] Duyen, T.T.M., H. Matsuura, K. Ujiie, M. Muraoka, et al.,Paper-based colorimetric biosensor for antibiotics inhibiting bacterial protein synthesis. Journal of Bioscience \& Bioengineering, 2016. 123(1): p. 96-100.

[55] Pellinen, T., T. Huovinen, and M. Karp, A cell-free biosensor for the detection of transcriptional inducers using firefly luciferase as a reporter. Analytical biochemistry, 2004. 330(1): p. 52-57.

[56] Kawaguchi, T., Y.P. Chen, R.S. Norman, and A.W. Decho,Rapid screening of quorum-sensing signal $N$-acyl homoserine lactones by an in vitro cell-free assay. Appl. Environ. Microbiol., 2008. 74(12): p. 3667-3671.

[57] Miller, M.B. and B.L. Bassler, Quorum sensing in bacteria. Annual Reviews in Microbiology, 2001. 55(1): p. 165-199.

[58] Wen, K.Y., L. Cameron, J. Chappell, K. Jensen, et al., A cell-free biosensor for detecting quorum sensing molecules in P. aeruginosa-infected respiratory samples. ACS synthetic biology, 2017. 6(12): p. 2293-2301.

[59] Titus-Ernstoff, L., R. Troisi, E.E. Hatch, J.R. Palmer, et al.,Birth defects in the sons and daughters of women who were exposed in utero to diethylstilbestrol (DES). International journal of andrology, 2010. 33(2): p. $377-384$.

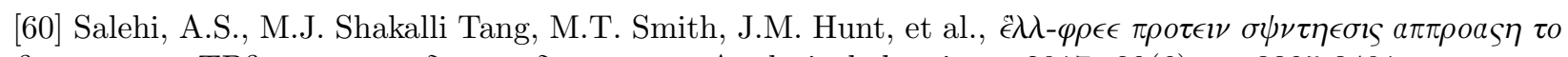
$\beta \imath o \sigma \epsilon \nu \sigma \imath \nu \gamma \eta T P \beta-\sigma \pi \epsilon \varsigma \imath \varphi \imath \varsigma \epsilon \delta \delta \circ \rho \imath \nu \epsilon \delta \imath \sigma \rho v \pi \tau o \rho \varsigma$. Analytical chemistry, 2017. 89(6): p. 3395-3401.

[61] Salehi, A.S., S.O. Yang, C.C. Earl, M.J.S. Tang, et al.,Biosensing estrogenic endocrine disruptors in human blood and urine: A RAPID cell-free protein synthesis approach. Toxicology and applied pharmacology, 2018. 345: p. 19-25.

[62] Gootenberg, J.S., O.O. Abudayyeh, J.W. Lee, P. Essletzbichler, et al., Nucleic acid detection with CRISPR-Cas13a/C2c2. Science, 2017. 356(6336): p. 438-442.

[63] Ilkhani, H. and S. Farhad, A novel electrochemical DNA biosensor for Ebola virus detection. Analytical biochemistry, 2018. 557: p. 151-155. 
[64] Jang, Y.-J., K.-H. Lee, T.H. Yoo, and D.-M. Kim,Interfacing a personal glucose meter with cell-free protein synthesis for rapid analysis of amino acids. Analytical chemistry, 2019. 91(3): p. 2531-2535.

[65] Soltani, M., B.R. Davis, H. Ford, J.A.D. Nelson, et al.,Reengineering cell-free protein synthesis as a biosensor: Biosensing with transcription, translation, and protein-folding.Biochemical Engineering Journal, 2018. 138: p. 165-171.

[66] Perez, J.G., J.C. Stark, and M.C. Jewett, Cell-free synthetic biology: engineering beyond the cell. Cold Spring Harbor perspectives in biology, 2016. 8(12): p. a023853.

[67] Karig, D.K., Cell-free synthetic biology for environmental sensing and remediation. Current opinion in biotechnology, 2017. 45: p. 69-75.

[68] Chen, F., J. Wang, L. Du, X. Zhang, et al., Functional expression of olfactory receptors using cell-free expression system for biomimetic sensors towards odorant detection. Biosensors and Bioelectronics, 2019. 130: p. 382-388.

[69] Daniels, B.C., Y.-J. Chen, J.P. Sethna, R.N. Gutenkunst, et al., Sloppiness, robustness, and evolvability in systems biology.Current opinion in biotechnology, 2008. 19(4): p. 389-395.

Table 1. The advantages of cell-free biosensors

\begin{tabular}{|c|c|c|}
\hline Features & Advantages & Reference \\
\hline Safety & $\begin{array}{l}\text { No GMOs will be released, and } \\
\text { therefore there is no biosafety } \\
\text { issue. }\end{array}$ & {$[48,66]$} \\
\hline Toxicity tolerance & $\begin{array}{l}\text { The system can operate in the } \\
\text { presence of toxins. }\end{array}$ & {$[67]$} \\
\hline Response time & $\begin{array}{l}\text { The tested materials can react } \\
\text { directly with the sensors in a } \\
\text { cell-free environment to } \\
\text { generate signals more quickly. }\end{array}$ & \\
\hline Selectivity & $\begin{array}{l}\text { It has the selectivity based on } \\
\text { biosensor elements, which can } \\
\text { reduce the interference with } \\
\text { other signals. }\end{array}$ & {$[51]$} \\
\hline Stability & $\begin{array}{l}\text { Cell-free systems have high } \\
\text { long-term storage stability by } \\
\text { the freeze-drying technology. }\end{array}$ & \\
\hline Sensitivity (Detection limit) & $\begin{array}{l}\text { The detection sensitivity is high; } \\
\text { the detection limit is low and can } \\
\text { even reach the nanomole level. }\end{array}$ & {$[68]$} \\
\hline Robustness & $\begin{array}{l}\text { Cell-free system can keep its } \\
\text { structure and function stable } \\
\text { when it is disturbed by } \\
\text { uncertain factors. }\end{array}$ & {$[69]$} \\
\hline Engineering operation & $\begin{array}{l}\text { Metabolic pathways can be } \\
\text { simplified and optimized by } \\
\text { adding different concentrations } \\
\text { of DNA components or by } \\
\text { mixing different cell extracts. }\end{array}$ & {$[55]$} \\
\hline
\end{tabular}

Figure legends 
Fig. 1. The workflow of cell-free biosensors.

Fig. 2. Recognition-response mechanisms of cell-free biosensor systems. (A) After the ligand is identified, reporter genes can be activated by binding activated transcription factors or releasing inhibitory transcription factors. Unknown ligands can be converted into detectable ligands by using metabolic enzymes before the identification. (B) Cell-free biosensors use NASBA-CRISPR/Cas9 cleavage system to identify different strains of virus. (C) Nucleic acid ligands are used to identify thrombin. When thrombin is absent, the reporter protein is successfully expressed, but the translation is blocked in the presence of thrombin. (D) When samples containing amino acids are added, incomplete CFPS is activated, leading to successful expression of the reporter protein.

Fig. 3. Signal output of cell-free biosensor systems. (A) The reporter gene $g f p$ is expressed in the CFPS system and output in the form of optical signal. (B) The reporter gene luc is expressed in the CFPS system and output in the form of optical signal. (C) The reporter gene lacZ is expressed in a paper-based cell-free system, and the color change on the measured paper can be easily detected by the naked eye. (D) The biosensor based on the cell-free genetic circuit captures the analyte through the nanostructured microelectrode to generate the electrochemical signal output.

Fig. 4. The applications of cell-free biosensors. 


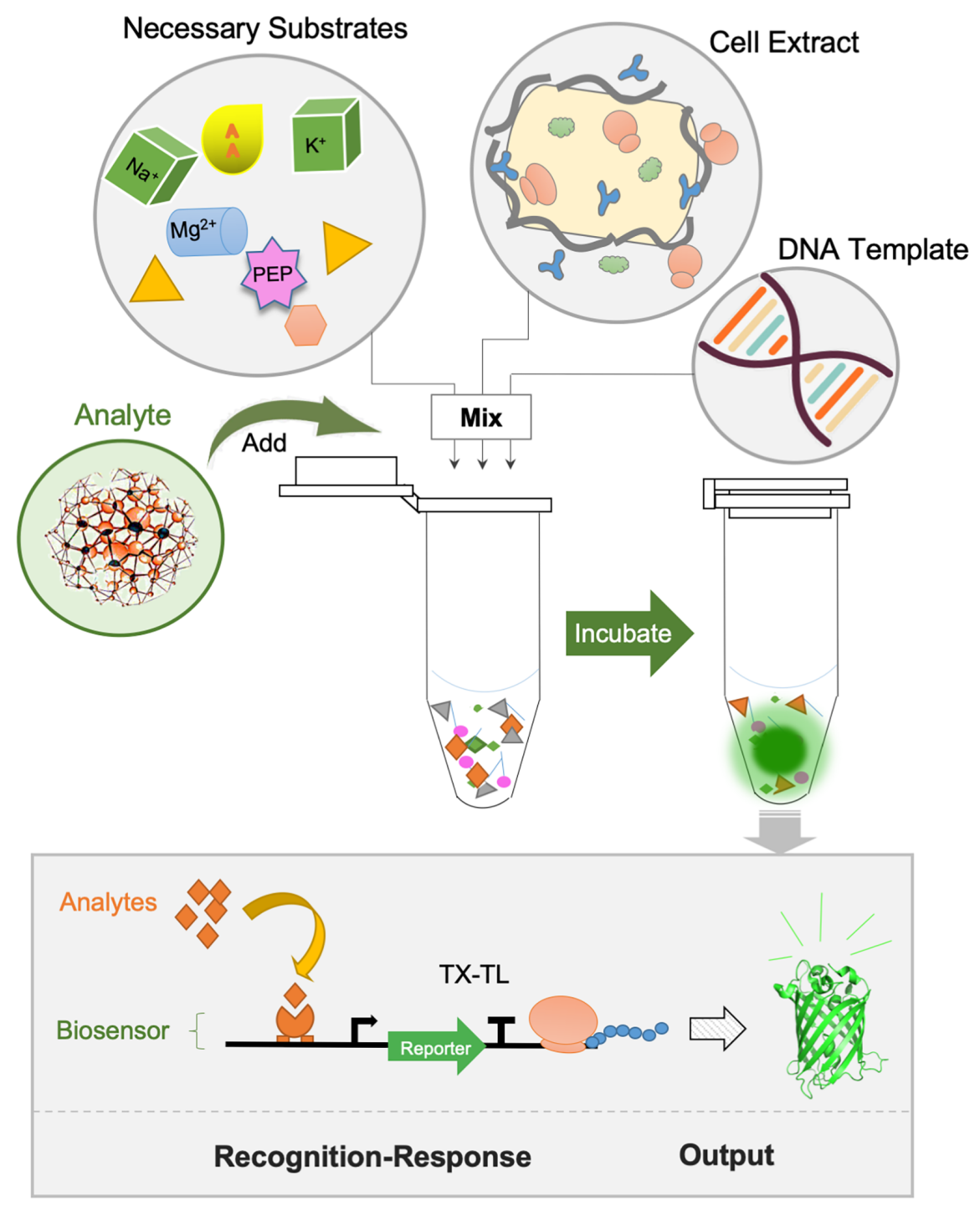


A Transcription factor activation/repression

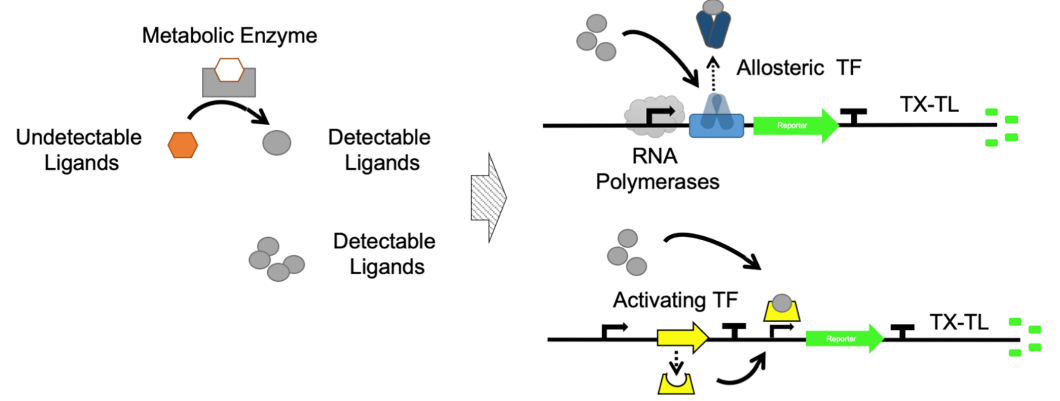

B Toehold switch and CRISPER-Cas recognition

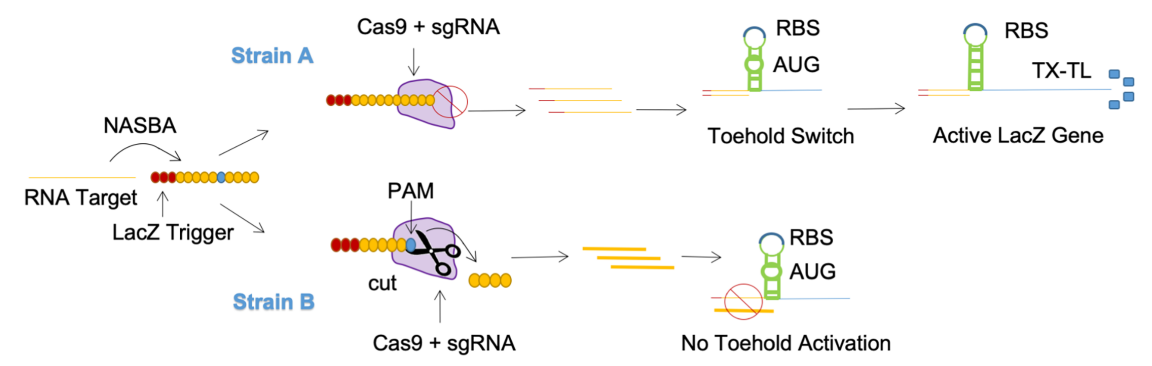

C Aptamer recognition
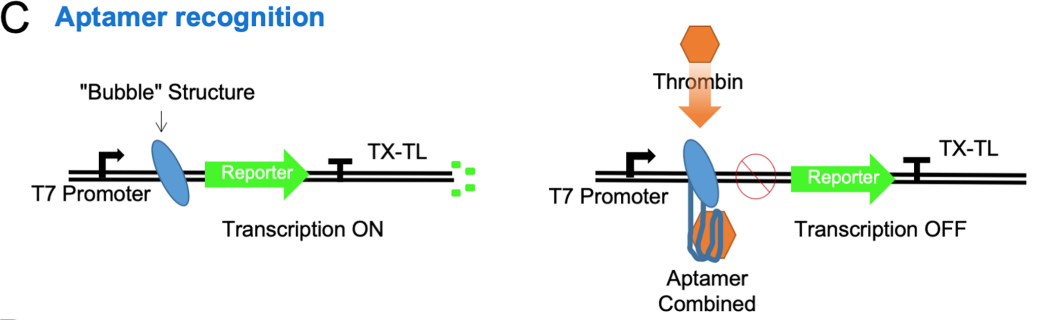

D CFPS component identification
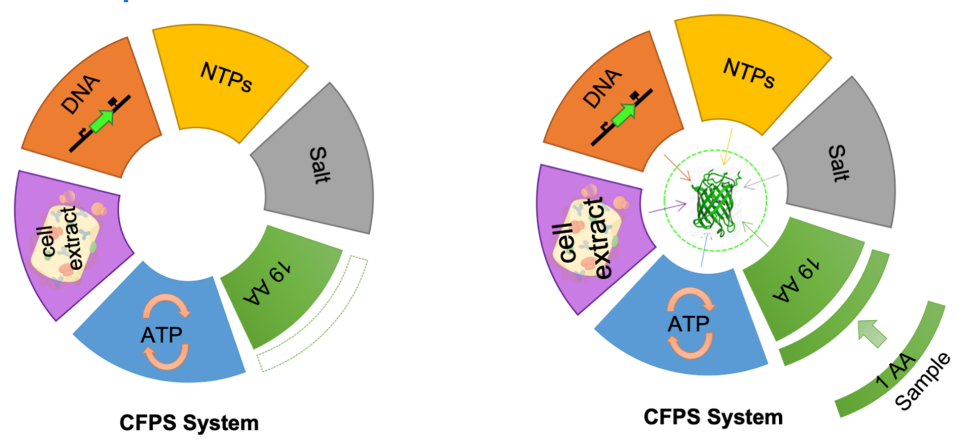
A

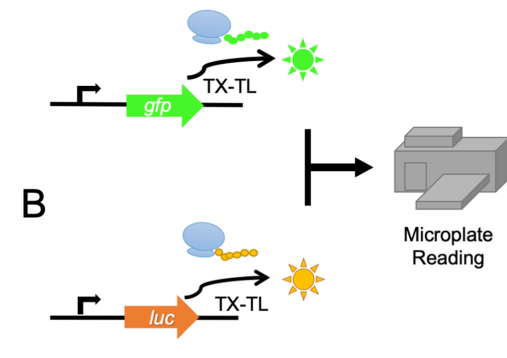

C
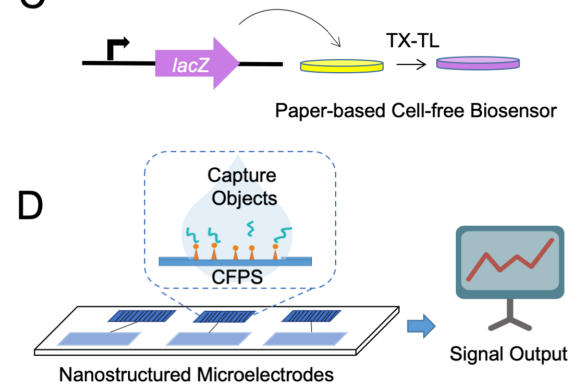

Nanostructured Microelectrodes

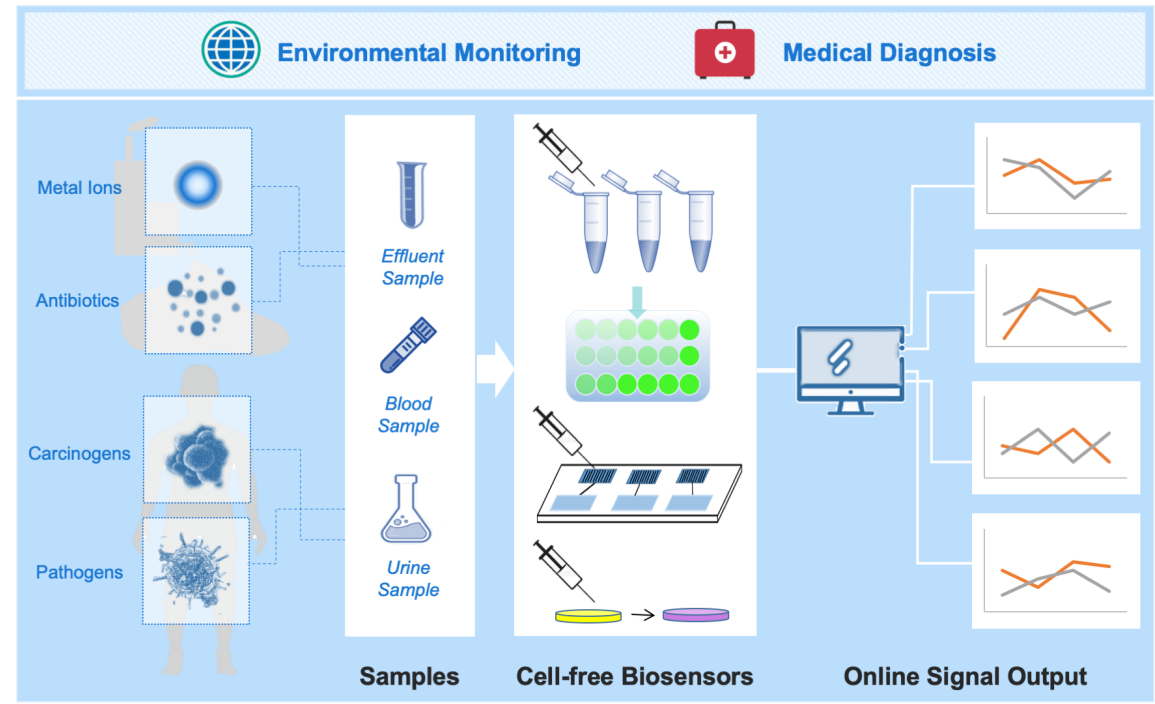

